\title{
Abscess rate of patients with penetrating abdominal injury in Zaria
}

\author{
Jerry Godfrey Makama*, Ekundayo Stephen Garba
}

Department of Surgery, Ahmadu Bello University Teaching Hospital, Shika-Zaria, Nigeria;

*Corresponding Author: jerlizabeth@yahoo.com

Received 20 February 2013; revised 23 March 2013; accepted 8 April 2013

Copyright (c) 2013 Jerry Godfrey Makama, Ekundayo Stephen Garba. This is an open access article distributed under the Creative Commons Attribution License, which permits unrestricted use, distribution, and reproduction in any medium, provided the original work is properly cited.

\section{ABSTRACT}

Background: Penetrating abdominal injury occurs when a foreign object pierces the skin. The morbidity and mortality associated with penetrating abdominal trauma is related to the intraabdominal complications. This study is, therefore, intended to determine the abscess rate resulting from penetrating abdominal trauma in Ahmadu Bello University Teaching Hospital Zaria. Method: A 6-year (January 2006-December 2011) retrospective study of penetrating abdominal trauma emphasizing on the rate of development of intra-abdominal abscess. Information was obtained from patients' case notes, operating room log books and surgical audit data. Information extracted included cases of penetrating abdominal trauma, intra-operative findings and cases of intra-abdominal abscesses. Results: A total of 39 cases of penetrating abdominal trauma were treated within this period of six years. $3(7.7 \%)$ were treated in 2006, $6(15.4 \%)$ in 2007, $3(7.7 \%)$ in 2008 and $11(28.2 \%)$ in 2009, $8(20.5 \%)$ in 2010, $9(23.1 \%)$ in 2011. 26 (66.7\%) were adult while $13(33.3 \%)$ were paediatric cases. The male were $37(94.9 \%)$ and the female were $2(5.1 \%)$, with a sex ratio of 18.5:1 (male to female). The age range was 5 - 60 years (39.92 mean). The commonest cause of injury was road traffic accident. At exploration, 13 (33.3\%) had organ injury only, 17 (43.6\%) suffer from both organ injury and intra-peritoneal emorrhage, 9 (23.1\%) had retroperitoneal haemorrhage only. The intraabdominal abscess rate of penetrating abdominal injury in Zaria was found to be $2.6 \%$. Conclusion: Contamination from either foreign object or organ injury is found to increase the risk of post-operative intra-abdominal abscess.
In this center, the abscess rate of penetrating abdominal trauma is comparatively low.

Keywords: Abscess; Penetrating; Abdominal Injury; Rate

\section{INTRODUCTION}

Penetrating abdominal injury occurs when a foreign object pierces the skin [1]. When the skin is pierced with a breach of the deep fascia, patient may require exploratory laparotomy to ensure the extend, or absence of injury to internal organs. However, the external appearance of the penetrating wound does not determine the extent of internal injuries. It is necessary to determine the trajectory of a penetrating wound. Also, one needs to consider all possible internal injuries. The mortality associated with penetrating abdominal trauma is related to the intra-abdominal complications such as organs injury, with refractory haemorrhagic shock [1,2]. Also, surgical site infections (SSIs), such as wound infection, fascial dehiscence, and intra-abdominal abscess commonly occur following penetrating abdominal trauma [3]. Colon wounds are recognized to be highly associated with intra-abdominal abscess (IAA) following penetrating abdominal trauma, whereas gastric wounds are thought to contribute minimally to abscess because of the bactericidal effect of low $\mathrm{pH}$ [3-5]. This study is, therefore, intended to determine the abscess rate of penetrating abdominal trauma in Ahmadu Bello University Teaching Hospital Zaria.

\section{MATERIAL AND METHODS}

This was a retrospective study done at Ahmadu Bello University Teaching Hospital Shika Zaria. All Patients, regardless of age that presented with penetrating abdominal trauma from January 2006 to December 2011 
were reviewed retrospectively, using patients' case notes, operating theatre log books and surgical audit data. For the purpose of this study, penetrating abdominal injury was defined as penetrating injury to the abdomen that has breached the deep fascia. Intra-operative findings such as evidence of pre peritoneal haematoma, peritoneal bruise/ tear, free fluid/blood in the peritoneal cavity including the pelvis, intra-abdominal organ injury, retroperitoneal haematoma and/or organ injury following penetrating abdominal trauma were noted. Those who had blunt abdominal injury were excluded. Also excluded were known diabetics, those on prolonged steroid therapy and immune-compromised patients. All the cases that develop intra-abdominal abscesses following surgery for penetrating abdominal injury were determined. The number of abscesses, the volume, sites, consistency and microbiology were also determined. Statistical data analysis was carried out using SPSS version 17.5. Simple frequencies were determined for variables. Where multiple responses were required, each response was treated as a separate variable. The critical level was set at 0.05 for all statistical tests.

\section{RESULT}

A total of 39 cases of penetrating abdominal trauma were treated within this period of six years. $3(7.7 \%)$ were treated in 2006, 6 (15.4\%) in 2007, 3 (7.7\%) in 2008 and 11 (28.2\%) in 2009, 8 (20.5\%) in 2010, 9 (23.1\%) in 2011. The general post election crisis in Nigeria particularly, in the northern region may have accounted for the unusually high number of cases in 2009. The commonest cause of penetrating abdominal trauma was Vehicular injury from road traffic accident (Figure 1). 26 (66.7\%) were adult while $13(33.3 \%)$ were paediatric cases. The male were $37(94.9 \%)$ and the female were 2 (5.1\%), with the sex ratio of 18.5:1 (male to female). The age range was 5 - 60 years (39.92 mean). The majority of the patients sustained anterior penetrating abdominal injury (Figure 2). At exploration, 13 (33.3\%) had organ injury only, 17 suffer from $(43.6 \%)$ both organ injury and intra-peritoneal haemorrhage, 9 (23.1\%) had retroperitoneal haemorrhage only. Among the 13 that had organ injury, Spleen was the highest injured organ (Figure 3). An average of $4 \mathrm{~L}$ of warm normal saline was used to lavage the intra-abdominal cavity of 36 patients while 3 were lavaged using 2 L of diluted povidone iodine. Among this three, two had faecal contamination of the abdominal cavity resulting from (L) colonic injury and the other had extensive gunshot injury to the abdomen. Thirty seven had tube drain inserted following laparotomy while two did not have drain. Among the thirty seven adult cases that had tube drain in place following surgery, only one developed an intraabdominal abscess 5 days after exploratory laparotomy. Reexplo-

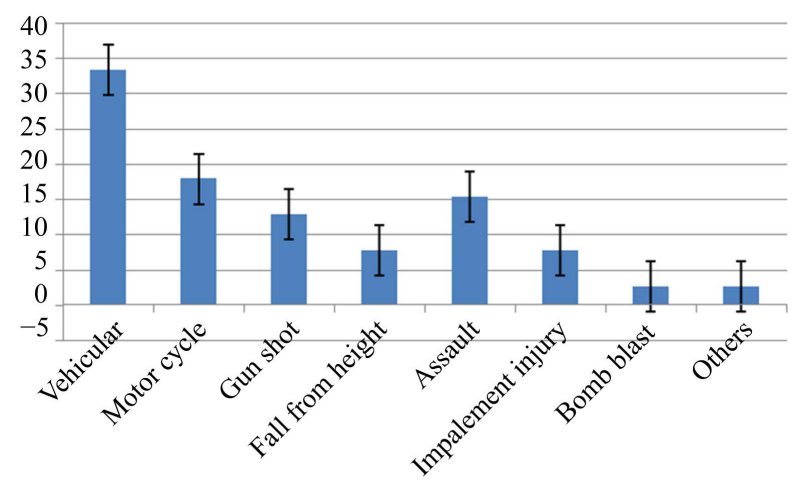

Figure 1. The common causes of penetrating abdominal trauma.

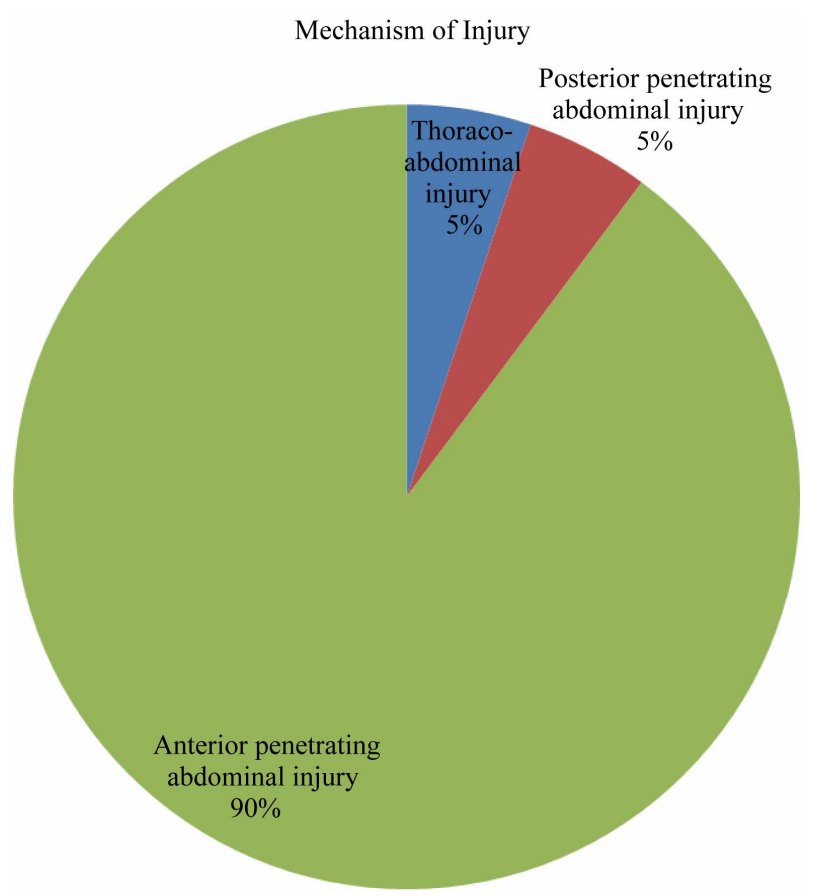

Figure 2. Mechanism of injury.

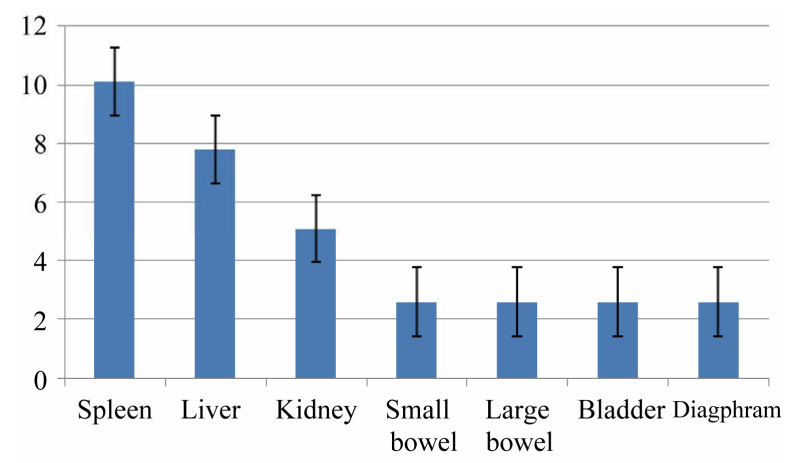

Figure 3. Organ injury.

ration revealed an abscess located in the (R) subphrenic space. About $315 \mathrm{ml}$ of pus was drained and a lavage with $4 \mathrm{~L}$ of warm normal saline done. A drain was then 
left in situ postoperatively. It was removed 5 days later when it has finished serving its purpose. Patient did well and was discharged home. Bacteriology of the abscess revealed mix microbiology. Both E. coli and anaerobes were cultured. The abscess rate of penetrating abdominal injury in Zaria was, therefore, computed and was found to be $2.6 \%$.

\section{DISCUSSION}

Emergency practitioners routinely encounter patients who suffer from abdominal trauma, being blunt or penetrating. Trauma is a physical injury caused by transfer of energy to and within the person involved $[6,7]$. Closerange injuries carry more kinetic energy than those sustained at a distance [6]. Thus, injuries sustained from closed-range kinetic energy are usually substantial, exceptionally deeper and severer than it thought to be [8]. Abdominal trauma is best categorized by a mechanism as blunt and penetrating abdominal injuries. The effect of the trauma could be mild in which case, the skin is only bruised, and the peritoneum is not breached. Thus, the injury is considered blunt compared to a particularly serious one where the peritoneum is not only breached or pierce but the intra-abdominal organ may be involved from varying degree to a shattered state or unsalvageable state. In this case, it is considered to be penetrating abdominal trauma. In penetrating abdominal trauma, the agent carries with it, into the intra-abdominal cavity debris and a contaminated trajectory. This is considered as an extremely high risk to the development of intra-abdominal abscess in these patients [9]. Other risk factors to develop intra-abdominal abscess in penetrating abdominal trauma including deposition of foreign body in the peritoneal cavity, large bowel perforation with spillage of faecal matter [9,10]. Individual risk factors such as age and presence of co-morbidity like diabetes mellitus are possibly useful but not well defined [9].

The mechanism of injury and the agent of injury influence the diagnostic work-up and the subsequent management. The anatomic site of injury and weapon (i.e. gun, knife) guide the diagnostic process [11]. Information such as the number of gunshots or the number of times the patient was stabbed, and the patient's position at the time of injury help describe the trajectory or path of the injuring object and subsequently, the associated organ injury $[11,12]$. The complications the patient may develop may be suspected also by the knowledge of the mechanism of injury.

Abdominal trauma patients are often difficult to assess clinically. Confounding factors, such as associated extraabdominal injuries or altered mental status (either from a head injury or intoxication), further complicate the evaluation. These confounding factors and lack of historical facts may represent different challenges in management of penetrating abdominal trauma. It is necessary to determine the trajectory of a penetrating wound. Also, it is necessary to consider all possible internal injuries [12]. Hepatic injury is considered to be the most common intra-abdominal organ injury. However, in this series, the commonest organ injury was splenic injury. The small bowel is injured more with penetrating than with blunt abdominal trauma, and it is the most commonly injured organ with penetrating abdominal trauma. However, in this series, small bowel injury was unusually small.

The introduction and refinement of diagnostic procedures and imaging studies, including peritoneal lavage, laparoscopy, computed tomography (CT), and focused ultrasonography, have reduced exploratory laparotomy and have directed the evolution of penetrating abdominal trauma care $[13,14]$. Technologies allow less invasive and more rapid and specific diagnostic evaluations [15, 16]. Thus, limit the number of exploratory laparotomy. However, the high cost and non availability of these imaging techniques in most centers including this center may have contributed to the high number of exploratory laparotomy.

The development of infection in trauma patients depends on the interaction of various factors such as prior health conditions, lesions caused by the injury and their complications, and, finally, the treatment modality [4,5, 17]. Traditionally, intra-abdominal abscesses are thought to occur after intra-abdominal surgical procedures, perforation of a hollow viscus following abdominal trauma [5]. Earlier investigators on abdominal trauma have proposed that an introduction of exogenous aerobic bacteria, such as staphylococci, from skin and foreign matter into the peritoneal cavity or other tissues usually increase the risk of development of intra-abdominal abscess [4,6-8]. On the other hand, clinical and microbiological studies have demonstrated that intestinal perforation and peritoneal contamination by endogenous aerobic and anaerobic microorganisms are the primary determinants for development of post-operative infection following abdominal trauma $[9,10]$. However, the only case that developed an intra-abdominal abscess in this review period did not suffer bowel injury with intestinal spillage.

In general, significantly reduced rates of abdominal infection regardless of the surgical techniques [18] or antibiotic protocols used in such patients with abdominal trauma without evidence of intestinal spillage have been noted by previous studies $[4,11,19]$. It is also true that timely intervention, sound surgical judgment, and skillful operative technique have been noted to be critical in reducing infection following trauma $[9,11]$. The routine lavage with normal saline may have contributed immensely to the low rate of abdominal abscess in this environment.

In addition, factors such as the severity and number of 
organs injured, amount of bacterial contamination, blood loss, therapeutic delay, and choice of antibiotics may significantly affect the outcome of treatment [5]. However, this was not a noticeable trend in this series.

The location of the abscess does not necessarily depend upon the area of the original injury or upon the surgical procedure. It is quite possible for a person to get a right subphrenic abscess following a left lower quadrant injury, perforation, or surgery [20]. The most popular sites of intra-abdominal abscesses are the subphrenic spaces, the perihepatic area (including the lesser sac), the lateral gutters, and the pelvis. The mechanism in the most common situation has been described as a patient stabbed in the abdomen may suffer a perforation of the intestines. The contents of the intestines then contaminate the peritoneal cavity, and, following surgical repair of the lacerated bowel, some contamination of the peritoneal cavity may remain. This, in turn, may result in an abscess in the pelvis, in the subphrenic spaces, beneath the liver, or in the lateral gutters [20].

Because intra-abdominal abscesses most often result from perforation of a hollow viscus or a subsequent surgical procedure, they potentially contain all of the organisms found in the alimentary tract [19]. Thus, it is often not possible to determine exactly which organisms are present, and one must, therefore, consider that any or all of the organisms normally found in the alimentary tract may play a role in the sepsis. If cultures are obtained at the time of the initial operative procedure, they will prove to be of inestimable value. Mixed flora (aerobes and anaerobes) are recovered from patients with intraabdominal abscesses at least five times more often than are either aerobes or anaerobes alone [20].

Identification of risk factors predictive of post-operative infection can influence surgical decisions [21,22], resulting in lower infection rates and reduction of costs. Examples of relevant areas in which cost-effective alterations in treatment are possible include selection of appropriate antibiotics, duration of antibiotic administration, treatment of specific abdominal injuries, meticulous operative techniques and wound care.

Copious irrigation of the peritoneal cavity with warm saline will naturally reduce residual soilage. This has shown to reduce the high rate of intra-abdominal infections. In cases of gross contamination or high risk for infection, the abdominal wound should be irrigated with saline too, packed opened, and closed secondarily.

\section{REFERENCES}

[1] Feliciano, D.V. and Rozcyki, G.S. (1995) The management of penetrating abdominal trauma. Advances in Surgery, 28, 1-39.

[2] Isenhour, J.L. and Marx, J. (2007) Advances in abdominal trauma. Emergency Medicine Clinics of North America, 25, 713-733. doi:10.1016/j.emc.2007.06.002

[3] Deitch, E.A., Livingston, D.H. and Hauser, C.J. (1999) Septic complications in the trauma patient. New Horizons, 7, 158-172.

[4] Hasper, D., Schefold, J.C. and Baumgart, D.C. (2009) Management of severe abdominal infections. Recent $\mathrm{Pa}$ tents on Anti-Infective Drug Discovery, 4, 57-65. doi:10.2174/157489109787236265

[5] Dente, C.J., Tyburski, J., Wilson, R.F., Collinge, J., Steffes, C. and Carlin, A. (2000) Ostomy as a risk factor for posttraumatic infection in penetrating colonic injuries: Univariate and multivariate analyses. Journal of Trauma, 49, 628-637.

[6] Nicholas, J.M., Rix, E.P., Easley, K.A., et al. (2003) Changing patterns in the management of penetrating abdominal trauma: The more things change, the more they stay the same. Journal of Trauma, 55, 1095-1108. doi:10.1097/01.TA.0000101067.52018.42

[7] Cotton, B.A. and Nance, M.L. (2004) Penetrating trauma in children. Seminars in Pediatric Surgery, 13, 87-97. doi:10.1053/j.sempedsurg.2004.01.004

[8] Maurer, E. and Morris Jr., J.M. (2004) Injury severity scoring in trauma. McGraw-Hill Companies, New York, 87-91.

[9] Fariñas-Alvarez, C., Fariñas, M.C., Fernandez-Mazarrasa, C., Llorca, J., Casanova, D. and Delgado-Rodríguez, M. (2000) Analysis of risk factors for nosocomial sepsis in surgical patients. British Journal of Surgery, 87, 10761081.

[10] Eberhardt, J.M., Kiran, R.P. and Lavery, I.C. (2009) The impact of anastomotic leak and intra-abdominal abscess on cancer-related outcomes after resection for colorectal cancer: A case control study. Diseases of the Colon \& Rectum, 52, 380-386. doi:10.1007/DCR.0b013e31819ad488

[11] Morales, C.H., Villegas, M.I., Villavicencio, R., González, G., Pérez, L.F., Peña, A.M. and Vanegas, L.E. (2004) Intraabdominal infection in patients with abdominal trauma. Archives of Surgery, 139, 1278-1285.

[12] Varin, D.S., Ringburg, A.N., Van Lieshout, E.M., Patka, P. and Schipper, I.B. (2009) Accuracy of conventional imaging of penetrating torso injuries in the trauma resuscitation room. European Journal of Emergency Medicine, 16, 305-311. doi:10.1097/MEJ.0b013e32832c3ab9

[13] DeMaria, E.J., Dalton, J.M., Gore, D.C., Kellum, J.M. and Sugerman, H.J. (2000) Complementary roles of laparoscopic abdominal exploration and diagnostic peritoneal lavage for evaluating abdominal stab wounds: A prospective study. Journal of Laparoendoscopic \& Advanced Surgical Techniques Part A, 10, 131-136. doi:10.1089/lap.2000.10.131

[14] Udobi, K.F., Rodriguez, A., Chiu, W.C. and Scalea, T.M. (2001) Role of ultrasonography in penetrating abdominal trauma: A prospective clinical study. Journal of Trauma, 50, 475-479. doi:10.1097/00005373-200103000-00011

[15] Martinez, M., Briz, J.E. and Carillo, E.H. (2001) Video thoracoscopy expedites the diagnosis and treatment of pe- 
netrating diaphragmatic injuries. Surgical Endoscopy, 15, 28-32. doi:10.1007/s004640002090

[16] Neal, M.D., Peitzman, A.B., Forsythe, R.M., et al. (2011) Over reliance on computed tomography imaging in patients with severe abdominal injury: Is the delay worth the risk? Journal of Trauma, 70, 278-284. doi:10.1097/TA.0b013e31820930f9

[17] Oberholzer, A., Keel, M., Zellweger, R., Steckholzer, U., Trentz, O. and Entel, W. (2000) Incidence of septic complications and multiple organ failure in severely injured patients is sex specific. Journal of Trauma, 48, 932-937.

[18] Kimura, T., Shibata, M. and Ohhara, M. (2005) Effective laparoscopic drainage for intra-abdominal abscess not amenable to percutaneous approach: Report of two cases. Diseases of the Colon \& Rectum, 48, 397-399. doi:10.1007/s10350-004-0846-y

[19] Delgado Jr., G., Barletta, J.F., Kanji, S., Tyburski, J.G., Wilson, R.F. and Devlin, J.W. (2002) Characteristics of prophylactic antibiotic strategies after penetrating abdominal trauma at a level I urban trauma center: A comparison with the East guidelines. Journal of Trauma, 53, 673-678. doi:10.1097/00005373-200210000-00009

[20] Hasper, D., Schefold, J.C. and Baumgart, D.C. (2009) Management of severe abdominal infections. Recent $\mathrm{Pa}$ tents on Anti-Infective Drug Discovery, 4, 57-65. doi:10.2174/157489109787236265

[21] Alzamel, H.A. and Cohn, S.M. (2005) When is it safe to discharge asymptomatic patients with abdominal stab wounds? Journal of Trauma, 58, 523-525. doi:10.1097/01.TA.0000152987.21630.39

[22] Adams, J.M., Hauser, C.J., Livingston, D.H., et al. (2001) The immunomodulatory effects of damage control abdominal packing on local and systemic neutrophil activity. Journal of Trauma, 50, 792-800. 